\title{
Identification, Expression Analysis of the Hsf Family, and Characterization of Class A4 in Sedum Alfredii Hance under Cadmium Stress
}

\author{
Shuang-Shuang Chen ${ }^{1,2,+}$, Jing Jiang ${ }^{1,2,+}$, Xiao-Jiao Han ${ }^{1,2}$, Yun-Xing Zhang ${ }^{1,2}$ and \\ Ren-Ying Zhuo 1,2,* (iD) \\ 1 State Key Laboratory of Tree Genetics and Breeding, Chinese Academy of Forestry, Beijing 100091, China; \\ chenshuang7876@sina.com (S.-S.C.); jiang2707@163.com (J.J.); hanxj@caf.ac.cn (X.-J.H.); \\ yunxingzh78@126.com (Y.-X.Z.) \\ 2 Key Laboratory of Tree Breeding of Zhejiang Province, The Research Institute of Subtropical of Forestry, \\ Chinese Academy of Forestry, Hangzhou 311400, China \\ * Correspondence: zhuory@gmail.com \\ + These authors contributed equally to this work.
}

Received: 22 January 2018; Accepted: 13 April 2018; Published: 17 April 2018

\begin{abstract}
Sedum alfredii Hance, a cadmium (Cd)/ zinc ( $\mathrm{Zn}) /$ lead $(\mathrm{Pb})$ co-hyperaccumulating species, is a promising phytoremediation candidate because it accumulates substantial amounts of heavy metal ions without showing any obvious signs of poisoning. The heat shock transcription factor (Hsf) family plays crucial roles in plant growth, development, and stress responses. Although the roles of some Hsfs in abiotic stress have been well studied in model plants, the Hsf family has not been systematically investigated in heavy metal hyperaccumulators. Here, we comprehensively analyzed the $\mathrm{Hsf}$ gene family in S. alfredii based on a transcriptome under $\mathrm{Cd}$ stress. There were $22 \mathrm{Hsf}$ members that were identified and phylogenetically clustered into three classes, namely, SaHsfA, $\mathrm{SaHsfB}$, and SaHsfC. All of the three classes shared similar motifs. The expression profiles of the $22 \mathrm{Hsf}$ members showed significant differences: $18 \mathrm{SaHsfs}$ were responsive to $\mathrm{Cd}$ stress, as were multiple SaHsp genes, including SaHsp18.1, SaHsp22, SaHsp26.5, SaHsp70, SaHsp90, and SaHsp101. Two class A4 members, SaHsfA4a and SaHsfA4c, exhibited transcriptional activation activities. Overexpression of SaHsfA4a and SaHsfA4c in transgenic yeast indicated an improved tolerance to Cd stress and $\mathrm{Cd}$ accumulation. Our results suggest $\mathrm{SaHsfs}$ play important regulatory roles in heavy metal stress responses, and provide a reference for further studies on the mechanism of heavy metal stress regulation by SaHsfs.
\end{abstract}

Keywords: Sedum alfredii Hance; heat shock transcription factor (Hsf); cadmium stress; class A4

\section{Introduction}

Abiotic stresses, such as heavy metal, heat, cold, drought, and salinity, adversely affect the growth and development of plants. They are defined by their negative impact on organisms in a given environment. Unlike animals, plants are sessile organisms. Therefore, they have had to develop defenses or adaptation mechanisms to deal with various stresses present during evolution [1-4]. These mechanisms can be processed including multiple genes and signaling pathways, which produce a series of physiological and biochemical changes in order to resist stress damage [5-7]. Each stage of these processes involves different types of transcription factors and cis-acting elements in stress-responsive promoters, which are controlled by different signal conditioning mechanisms in order to enable plant adaptation to environmental stresses [8]. Among these important transcription factors, heat shock transcription factors (Hsfs) are well known for responding to external high-temperature stress and 
activating the expression of heat shock proteins (Hsps) by combining with the heat shock element (HSE) in the thermal signal transduction pathway $[2,4,7]$.

Hsfs are part of an evolutionarily conserved gene family belonging to eukaryotes $[9,10]$. The structure and function of Hsfs are considerably conserved, despite the fact that there are only a few genes that undergo biological genome replication events, from the lower organism to the higher living of the large genome $[10,11]$. Almost all Hsfs have a conserved DNA-binding domain (DBD) containing three helices and four sheets. This domain, with hydrophobic amino acids, can form a special structure, activating the formation of Hsfs to bind with the HSE element of Hsp genes' promoter efficiently [7,10,12]. Similar to the DBD domain, the oligomerization domain (OD: HR-A/B regions) is a conserved domain located at the N-terminus of the Hsfs. Additionally, there are four other conserved structures, namely, the nuclear localization signal (NLS), nuclear export signal (NES), activator motif (AHA), and repression domain (RD). The Hsf gene family is divided into three classes based on the length of the flexible linker peptide between the DBD and HR-A/B, and the number of amino acids inserted between the HR-A and HR-B regions. Class A and B members contain AHA and RD motifs, respectively [7].

$H s f$ gene families are complex and large in higher plants. The size of the Hsf gene family varies greatly in the different plant species presented, with 21 members in the model plant Arabidopsis thaliana, 25 members in Oryza sativa, 28 members in Populus trichocarpa, 16 members in Medicago truncatula, 26 members in Glycine max, 25 members in Zea mays, and up to 56 members in Triticum aestivum [13-21]. Recently, many studies have demonstrated that the functions and characteristics of $H s f s$ differ among plant species in response to heat stress and other abiotic stresses [2,3,17,22-24]. Presently, two HsfA genes that are involved in cadmium (Cd) tolerance have been functionally characterized. HsfA1a from tomato (Solanum lycopersicum L. cv Ailsa Craig) induces an increase of melatonin levels under cadmium stress [25]. The HsfA4a gene in wheat (Triticum aestivum) and O. sativa has been reported to confer strong $\mathrm{Cd}$ tolerance [26]. These findings demonstrate the potential for research into heavy metal stress responses in the Hsf gene family.

Sedum alfredii Hance, a Cd/zinc $(\mathrm{Zn}) /$ lead $(\mathrm{Pb})$ co-hyperaccumulating species of Crassulaceae, was first discovered in an abandoned $\mathrm{Pb} / \mathrm{Zn}$ mine in Southeast China [27]. S. alfredii, a good material with perennial, asexual reproduction and a considerable biomass, can accumulate substantial amounts of heavy metal ions without displaying any obvious signs of poisoning. Thus, it is considered a promising candidate material in the field of phytoremediation. To date, several important physiological indices, including the accumulation capacity and translocation rates of different metal ions, have been characterized in this plant [28-30]. Additionally, some studies have been carried out on the molecular mechanism underlying the hyper-accumulation and hyper-tolerance to heavy metals in S. alfredii. Overexpression of the genes $S a M T 2, S a C u / Z n S O D$, and $S a R E F$, isolated from S. alfredii, conferred a higher tolerance to $\mathrm{Cd}$ stress in transgenic tobacco or $A$. thaliana [31-33]. Nevertheless, the Hsf family in $S$. alfredii has not yet been systematically investigated through phylogenetic, gene structure, conserved motif, and expression profiling analyses. Fortunately, the transcriptome data of S. alfredii were obtained [34], enabling the characterization of Hsf family members as well as their responses to heavy metal stress at the molecular level. In this study, bioinformatics and gene expression analyses were used to identify the $22 \mathrm{Hsf}$ family members in S. alfredii. We also performed the conserved domain, motif, and phylogenetic analyses. Additionally, the expression patterns of the SaHsf genes were profiled in response to heavy metal stress. Finally, the overexpression of two SaHsfA4 members was shown to enhance $\mathrm{Cd}$ tolerance in yeast. These results provide a foundation for further functional research on $\mathrm{Hsf}$ genes in S. alfredii and may serve as a reference for future studies on the mechanism of heavy metal stress regulation by SaHsfs. 


\section{Results}

\subsection{Twenty Two SaHsf Members were Identified and Classified into Three Classes}

To identify the Hsf genes in S. alfredii, a Hidden Markov Model (HMM) profile of the Hsf DBD domain (Pfam: PF00447) was used as a query against the $S$. alfredii transcriptome database. A total of 22 UniGenes containing DBD domains were obtained and annotated according to the Hsfs that were reported in other plants. Subsequently, the full coding regions of the candidate genes were cloned and sequenced. Finally, all of the SaHsf genes were verified using the online tools SMART and Pfam. Thereafter, the $22 \mathrm{Hsf}$ genes in S. alfredii were classified and named according to the rules of the Hsf families from A. thaliana and O. sativa and the HEATSTER tool (Table 1). The coding sequence (CDS) size for the SaHsfs ranged from 849 to $1563 \mathrm{bp}$, and the corresponding predicted protein lengths and molecular weights ranged from 282 to 520 amino acids and 31.99 to $57.42 \mathrm{kDa}$ (kilodalton), respectively (Table 1).

Table 1. The basic information list of the identified heat shock transcription factors (Hsfs) in S. alfredii.

\begin{tabular}{cccccc}
\hline Number & Gene Name & ORF Length (bp) & No. of AA & Mol. Wt. (kDa) & pI \\
\hline 1 & SaHsfA1a & 1482 & 493 & 55.11 & 4.66 \\
2 & SaHsfA1b & 1491 & 496 & 54.84 & 5.02 \\
3 & SaHsfA1d & 1563 & 520 & 57.42 & 4.65 \\
4 & SaHsfA2a & 1221 & 406 & 45.82 & 4.95 \\
5 & SaHsfA2b & 1122 & 373 & 42.69 & 4.43 \\
6 & SaHsfA3 & 1545 & 514 & 57.75 & 5.04 \\
7 & SaHsfA4a & 1332 & 443 & 50.10 & 5.36 \\
8 & SaHsfA4c & 1302 & 433 & 49.32 & 6.20 \\
9 & SaHsfA5 & 1284 & 427 & 47.53 & 5.45 \\
10 & SaHsfA6b & 1143 & 380 & 43.33 & 6.69 \\
11 & SaHsfA8 & 1263 & 420 & 48.63 & 4.89 \\
12 & SaHsfB1 & 855 & 284 & 31.83 & 4.50 \\
13 & SaHsfB2a & 1038 & 345 & 37.49 & 6.91 \\
14 & SaHsfB2b & 942 & 313 & 34.47 & 4.62 \\
15 & SaHsfB2c & 993 & 330 & 36.52 & 4.99 \\
16 & SaHsfB2d & 1047 & 348 & 37.61 & 5.35 \\
17 & SaHsfB2e & 849 & 282 & 31.99 & 7.89 \\
18 & SaHsfB2f & 1140 & 379 & 41.08 & 8.15 \\
19 & SaHsfB4a & 1032 & 343 & 39.57 & 6.74 \\
20 & SaHsfB4b & 885 & 294 & 33.81 & 7.47 \\
21 & SaHsfC1a & 930 & 309 & 35.11 & 5.45 \\
22 & SaHsfC1b & 873 & 290 & 32.64 & 6.73
\end{tabular}

Note: $\mathrm{ORF}$-open reading frame; No. of AA—number of amino acids; Mol. Wt-molecular weight; pI—isoelectric point.

\subsection{Phylogenetic Analysis of Hsfs in S. alfredii}

To examine the evolutionary characteristics of the Hsf proteins, a phylogenetic tree was constructed using the Hsf protein sequences from $S$. alfredii and the model plants A. thaliana and O. sativa. The full-length amino acid sequences of these Hsf proteins are shown in Supplementary File 1 and Table S1. According to the phylogenetic tree, the SaHsfs were grouped into three classes, namely, A, B, and C (Figure 1). Among them, class A was the largest subgroup with 11 Hsf proteins from seven subclasses (A1, A2, A3, A4, A5, A6, and A8), followed by class B with 9 members from three subclasses (B1, B2 and B4), while class C included only two members from subclass C1 (Figure 1, Table 2). Additionally, the class A members were divided into three smaller branches. Specially, the class A member SaHsfA3 had relatively distant evolutionary relationships with other class A members. Unlike class A3, the class A4 and A5 members had relatively close evolutionary relationships with class B members. No members were found in the A7 subclass and only one member was found in the B1 subclass, when we compared the numbers of each subclass in the SaHsf family with those in other plant species—such as A. thaliana [19], O. sativa [20], P. trichocarpa [18], and Z. mays [16] (Table 2). In addition, the B2 subclass included six members, while this subclass contained less than five members in other plant species (two members in A. thaliana, three members in $O$. sativa and $P$. trichocarpa, and four members in Z. mays). 


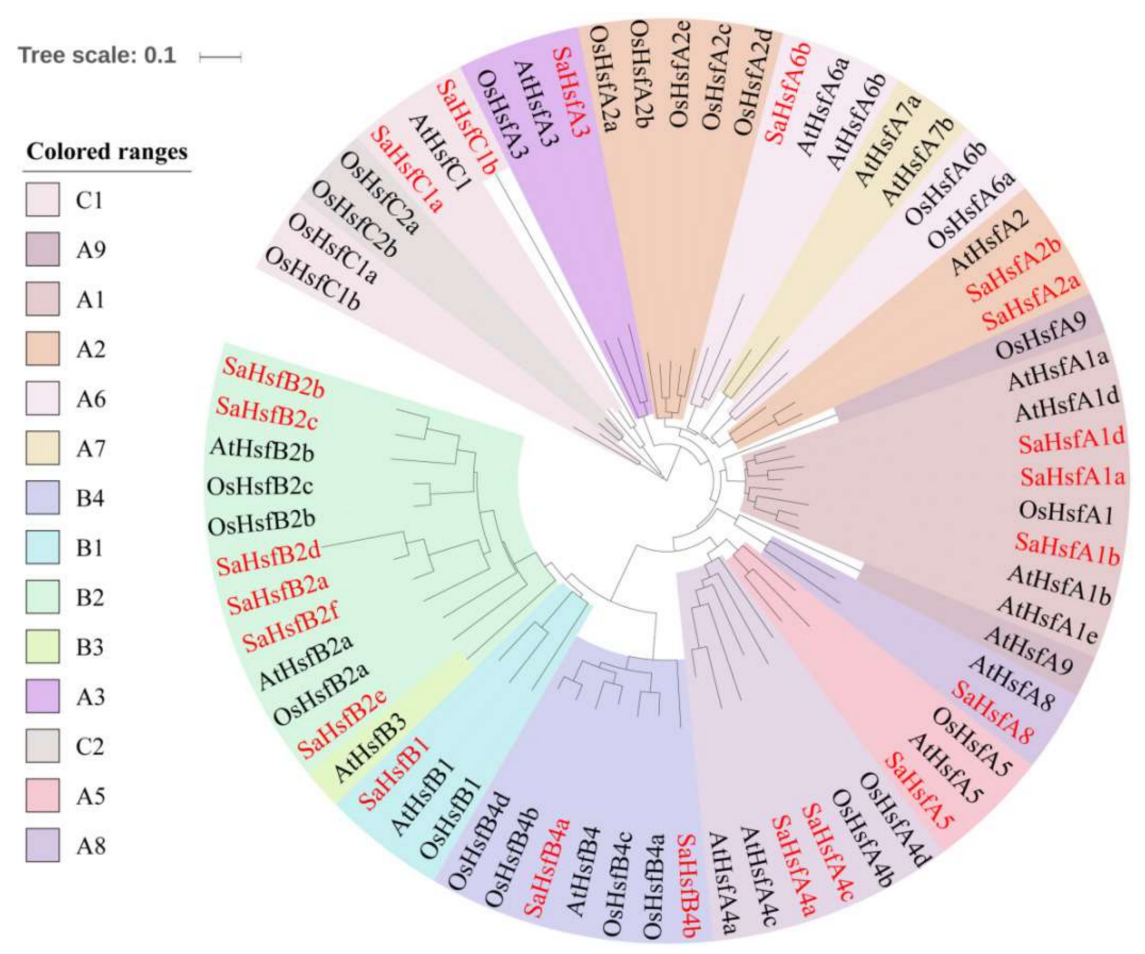

Figure 1. Phylogeny and distribution of heat shock transcription factor (Hsf) proteins. Phylogenetic tree of Hsf proteins from A. thaliana, O. sativa, and S.alfredii. The tree was generated with MEGA 6.0 software using the neighbor-joining (NJ) method. Hsfs in S.alfredii were labeled with the red color.

Table 2. Members of the Hsfs classes and subclasses in different plant species.

\begin{tabular}{|c|c|c|c|c|c|c|}
\hline Class & Subclass & A. thaliana & O. sativa & P. trichocarpa & Z. mays & S. alfredii \\
\hline \multirow{9}{*}{$\mathrm{A}$} & A1 & 4 & 1 & 3 & 2 & 2 \\
\hline & A2 & 1 & 5 & 1 & 2 & 3 \\
\hline & A3 & 1 & 1 & 1 & 1 & 1 \\
\hline & $\mathrm{A} 4$ & 2 & 2 & 3 & 3 & 2 \\
\hline & A5 & 1 & 1 & 2 & 1 & 1 \\
\hline & A6 & 2 & 2 & 2 & 2 & 1 \\
\hline & A7 & 2 & 0 & 2 & 2 & 0 \\
\hline & A8 & 1 & 0 & 2 & 2 & 1 \\
\hline & A9 & 1 & 1 & 1 & 0 & 0 \\
\hline \multirow{4}{*}{ B } & B1 & 1 & 1 & 1 & 2 & 1 \\
\hline & B2 & 2 & 3 & 3 & 4 & 6 \\
\hline & B3 & 1 & 0 & 2 & 0 & 0 \\
\hline & B4 & 1 & 4 & 4 & 1 & 2 \\
\hline \multirow{3}{*}{$\mathrm{C}$} & $\mathrm{C} 1$ & 1 & 2 & 1 & 2 & 2 \\
\hline & $\mathrm{C} 2$ & 0 & 2 & 0 & 1 & 0 \\
\hline & $\begin{array}{c}\text { Total } \\
\text { members }\end{array}$ & 21 & 25 & 28 & 25 & 22 \\
\hline
\end{tabular}

\subsection{Conserved Domains and Motifs in SaHsf Proteins}

The detailed knowledge available on the Hsf functional domains in the model plants A. thaliana and O. sativa enabled us to analyze the domains of the $22 \mathrm{SaHsfs}$. Six conserved domains, including the DBD, HR-A/B, NLS, AHA, RD, and NES, were predicted in the SaHsf family using the online tool HEATSTER (Table 3). The most conserved domains (DBD and HR-A/B) in Hsfs existed in all of the predicted SaHsf proteins. The DBD domain consisted of three helices and four sheets (Figure 2). However, SaHsfB2d and SaHsfC1b had no $\beta 3$ and $\beta 4$ sheets. Most of the SaHsf proteins included the NLS domain, except for $\mathrm{SaHsfB} 1$ and $\mathrm{SaHsfB} 2 \mathrm{~m}$. The NES domain was detected in two A subclasses (A1 and A4 members) and one B subclass members (SaHsfB4a). With the exception of SaHsfA1a and SaHsfA1d, all of the other class A members had AHA domains. However, this domain was not found in class B or C. All of the 
class B members had the RD domain, except for SaHsfB2e. The online tool MEME was used to search for motifs in the SaHsf proteins. There were 25 potential motifs that were found, the details of these motifs are given in Table 4. As shown in Figure 3B, motifs 1, 2, and 4 (inferred as DBD domain) were found in all of the 22 SaHsf members, however, motifs 2 and 4 were missing in SaHsfC1b. Different subclasses contained the same motifs and had their own unique motifs. In subclass B, motif 22 was only found in SaHsfB2 members, while motif 18 was found in SaHsfB1 and SaHsfB2 members, and motifs 19 and 20 were only discovered in SaHsfB4 members. Similarly, motifs 7, 9, and 11 were unique to SaHsfA members, while motifs 15 and 16 existed only in SaHsfA2 members. Moreover, SaHsfCla contained motif 6, which was also found in class A members. Notably, motif 3 was inserted between motif 2 and motif 4 in SaHsfA3, and motif 7 was also unique to this protein. As shown in Figure 3A, SaHsfA3 had a relatively distant relationship with other class A members. This result might have been related to the protein motifs. Generally, most of the closely related members had similar motif compositions.

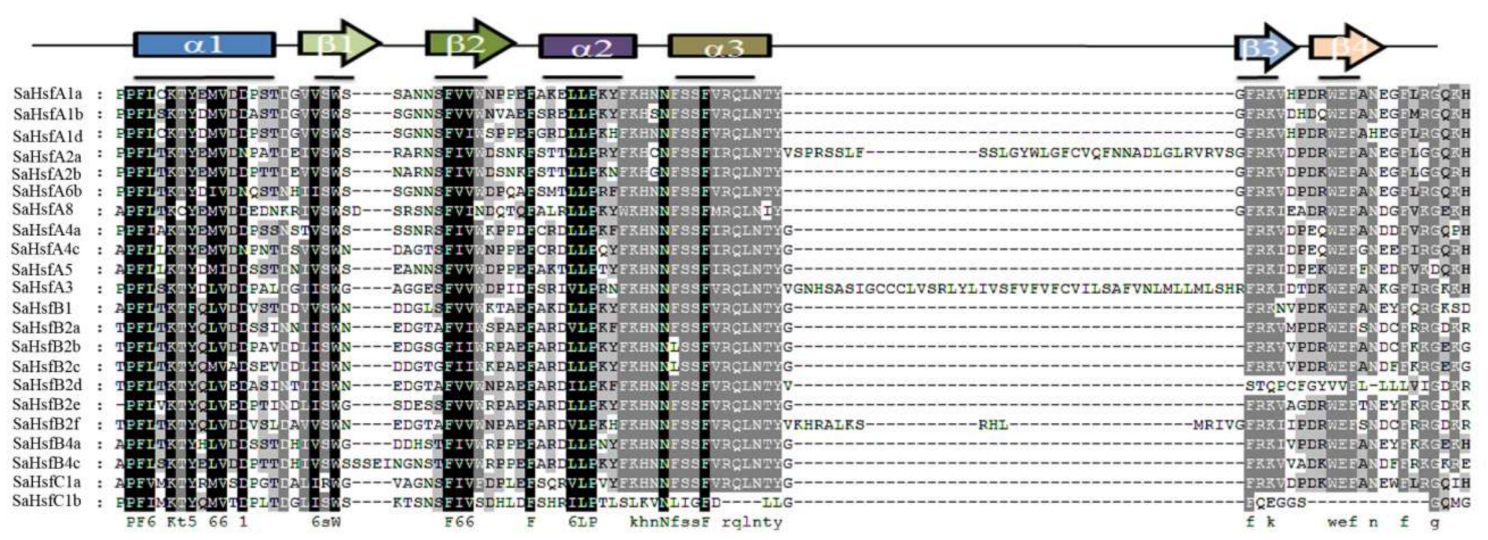

Figure 2. Multiple sequence alignment of the DNA-binding domain (DBD) of the Hsf protein family in S. alfredii. The different backgrouds, black and gray, indicated completely and partly conserved amino acids in proteins, respectively.

A

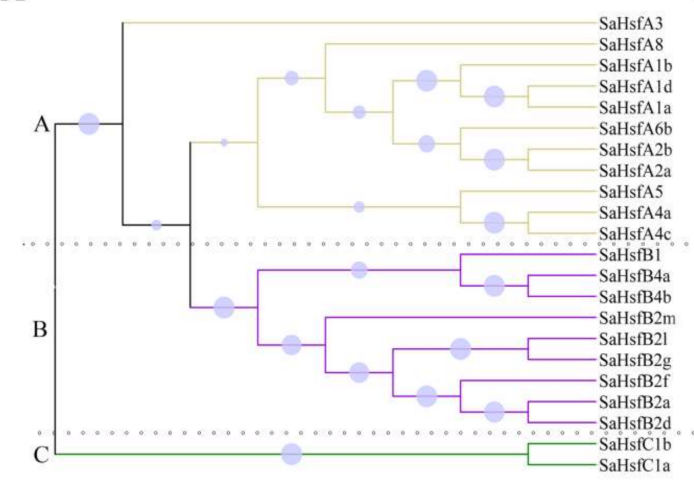

B

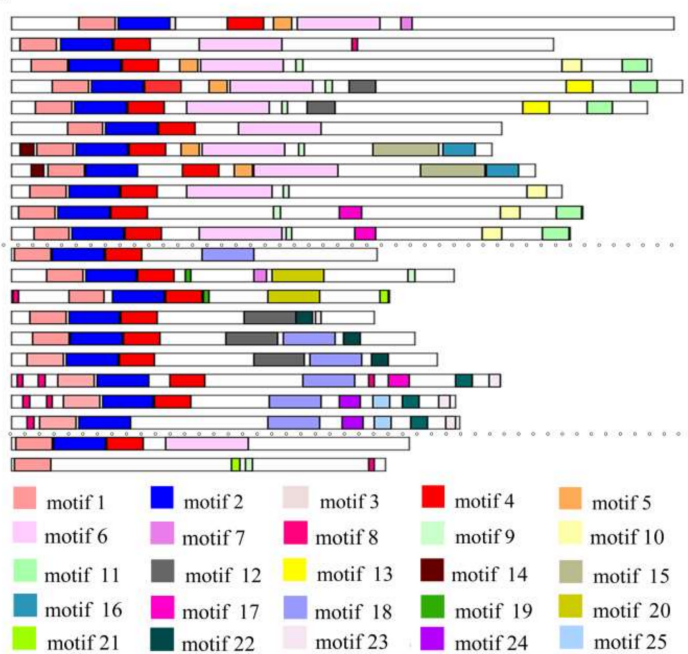

Figure 3. Phylogenetic relationships and motif compositions of the Hsf family in S. alfredii: (A) multiple alignment of Hsf proteins from S. alfredii was performed using MEGA 6.0 by the neighbor-joining (NJ) method with 1000 bootstrap replicates, which represented by circles; (B) schematic representation of each of the conserved motifs in the Hsf proteins was identified by the MEME online tool. Different motifs are represented by different colored boxes. The dashed lines are used to cluster and distinguish the genes into the A, B, and C classes. 
Table 3. Function domains found by HEATSTER in SaHsfs.

\begin{tabular}{|c|c|c|c|c|c|c|}
\hline \multirow{2}{*}{ Name } & \multicolumn{6}{|c|}{ Domains } \\
\hline & DBD & HR-A/B & NLS & AHA & RD & NES \\
\hline SaHsfA1a & 24-117 & 129-186 & 204-214 (RIIGENNKKRR) & & & 454-461 (ITDQMELL) \\
\hline SaHsfA1b & $20-113$ & 133-194 & 215-225 (RRITSSNKKRR) & (AHA2) 431-440 (DVFWEQFLST) & & 481-488 (LTSQMGLL) \\
\hline SaHsfA1d & $37-130$ & $156-220$ & 238-248 (RRINEANKKQR) & & & 488-495 (ITDQIGLL) \\
\hline SaHsfA2a & $33-159$ & 175-239 & 255-268 (RKALDGANVKRKRT) & (AHA1) 314-322 (SLLRAGLES) & & \\
\hline SaHsfA2b & $25-118$ & 134-198 & 214-228 (RKALDDAYSKRKRRL) & (AHA1) 313-320 (QMLWDELV) & & \\
\hline SaHsfA3 & $57-194$ & $222-268$ & 294-303 (KMKRKFITHH) & $\begin{array}{l}\text { (AHA3) 472-481 (FTDGWEFGSM) } \\
\text { (AHA4) 489-495 (LELGSPS) }\end{array}$ & & \\
\hline SaHsfA4a & $11-104$ & 130-187 & 205-208 (KKRR) & $\begin{array}{l}\text { (AHA1) 253-262 (ITHWEKIIYQ) } \\
\text { (AHA2) 383-392 (DTFWAQFLTE) }\end{array}$ & & 430-437 (LVEQMGHI) \\
\hline SaHsfA4c & 22-115 & 139-196 & 214-217 (KKRR) & $\begin{array}{l}\text { (AHA1) 264-273 (FSYWENILYS) } \\
\text { (AHA2) 369-378 (DVFWEQYLTE) }\end{array}$ & & 420-427 (ITEQMGQL) \\
\hline SaHsfA5 & 19-112 & 132-189 & 200-215 (TKINSMEFSAYSKKRR) & (AHA) 404-413 (DAFWEQYLTE) & & \\
\hline SaHsfA6b & $48-141$ & $162-227$ & 250-257 (AAANKRRH) & (AHA) 332-341 (QVFWEGFLNN) & & \\
\hline SaHsfA8 & $13-121$ & 154-211 & 326-334 (VDNTWYANH) & (AHA1) 312-321 (DDAILDHFIF) & & \\
\hline SaHsfB1 & $7-100$ & 150-187 & & & 255-261 (KLFGVWL) & \\
\hline SaHsfB2a & $45-138$ & 203-239 & 313-317 (KRART) & & 304-310 (KLFGFQL) & \\
\hline SaHsfB2b & $21-114$ & $170-206$ & 268-272 (KRVKR) & & 259-265 (KLFGVSI) & \\
\hline SaHsfB2c & $17-110$ & $191-227$ & 290-294 (KRRKK) & & 281-287 (MLFGVSI) & \\
\hline SaHsfB2d & $27-120$ & $202-238$ & 320-324 (KRARG) & & 311-317 (RLFGFQL) & \\
\hline SaHsfB2e & 19-112 & $177-213$ & & & & \\
\hline SaHsfB2f & $41-134$ & $229-265$ & 355-359 (KRARE) & & 346-352 (SLFGYQL) & \\
\hline SaHsfB4a & $32-125$ & 206-242 & 322-325 (SSSG) & & 312-318 (RLFGVPL) & 336-338 (NLM) \\
\hline SaHsfB4b & $49-146$ & $202-238$ & 286-289 (KRFH) & & 276-282 (KLFGVSI) & \\
\hline SaHsfCla & 8-102 & $127-170$ & 196-201 (DKRRRM) & & & \\
\hline SaHsfC1b & 7-101 & $113-156$ & 182-187 (NKRRRL) & & & \\
\hline
\end{tabular}


Table 4. Motifs identified by MEME tools.

\begin{tabular}{|c|c|c|}
\hline Motif & Width & Reference Sequences \\
\hline 1 & 29 & QRAAPPPFELTKTYQEMDDPSTDGIVSWSS \\
\hline 2 & 41 & GNSFVVWDPEFARDLLPKYFKHNNFSSFVRQLNTYGFRKV \\
\hline 3 & 6 & EGDCCC \\
\hline 4 & 29 & DPDRWEFANEGFLRGZKHLLKTIKRRKPI \\
\hline 5 & 15 & ACVEVGKYGLEEEVE \\
\hline 6 & 65 & LKPDKNVLMEJVKLKQZQQSSDKQLLMDRLQGMEQRQQQMMSFLAKAVQNPGFLSQFVQQQA \\
\hline 7 & 10 & HHPHLSGRSC \\
\hline 8 & 6 & SPPPPP \\
\hline 9 & 6 & NKKRRL \\
\hline 10 & 16 & TGVNDVFMWEQYLTEHP \\
\hline 11 & 21 & DKNQNINNITDQMGLLTSSAK \\
\hline 12 & 22 & GGQIVKYQPFLDDMPTFFRNMM \\
\hline 13 & 22 & FDIENIPPEHENTDGSAYDDVM \\
\hline 14 & 11 & IASEPIPRPME \\
\hline 15 & 51 & RAGLESDSHNNVDVQQPESVARIEESNLDSVARIEESNLDSVNZKLWDELLAGNLVJDNDDD \\
\hline 16 & 26 & QLLGDIPEAEDLEGQPSDWEEEDLQ \\
\hline 17 & 18 & HWEKIJYQIGRECGEDMF \\
\hline 18 & 6 & HHHHNN \\
\hline 19 & 41 & RAELMEENERLKKEINTQLTSELSHMKLCTNIYSMMSNYNP \\
\hline 20 & 8 & KRFHNNGH \\
\hline 21 & 14 & PKLFGVQIGSKRAR \\
\hline 22 & 41 & QDPGNPSMESKLQLDLLQSEKYLEEQGGSSGGNAGAMDEKEC \\
\hline 23 & 17 & VGCGSSNSSQAESMMNP \\
\hline 24 & 9 & DLLQLQQPG \\
\hline 25 & 14 & LLAAVVETQAIQAA \\
\hline
\end{tabular}

Note: Motif numbers corresponded to the motifs in Figure 3.

\subsection{Expression Profiles of SaHsfs}

To investigate the potential functions of the $\mathrm{SaHsf}$ genes under normal conditions, expression profiles of the SaHsf family members in S. alfredii were generated using quantitative real-time polymerase chain reaction (qRT-PCR) data from three tissues, including root, stem, and leaf (Figure 4). The expression patterns of most of the SaHsf genes were similar in different tissues. SaHsfA1b, SaHsfA4a, SaHsfA4c,

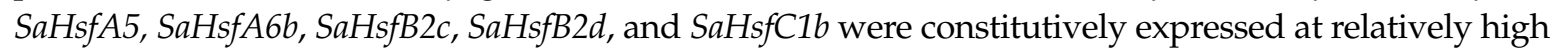
levels, while SaHsfA2a, SaHsfA2b, SaHsfB2a, SaHsfB2e, SaHsfB4b, and SaHsfC1a were expressed at low levels in all of the tested tissues. Some genes were expressed greatly in specific tissues. For example, the relative expression levels of $S a H s f B 2 f$ in the root and $S a H s f B 4 a$ in the stem were higher than in other tissues. In addition, SaHsfA8 and SaHsfB1 were detected only in the root, while SaHsfB4a was only expressed in the stem (Figure 4).

To examine the heavy metal response patterns of the SaHsfs in S. alfredii, we determined their expression levels in different tissues (root, stem, and leaf) under $\mathrm{CdCl}_{2}$ treatment. As a result of the properties of the experimental material (a $\mathrm{Cd} / \mathrm{Zn} / \mathrm{Pb}$ co-hyperaccumulating species), 11, 15, and $12 \mathrm{SaHsf}$ genes were responsive to heavy metal $(\mathrm{Cd})$ stress in the root, stem, and leaf, respectively (Figure 4). As shown in Figure 4A, SaHsfA2a, SaHsfA2b, SaHsfA5, SaHsfB2a, SaHsfB2e, and SaHsfC1a were up-regulated under $\mathrm{CdCl}_{2}$ treatment in the root. Conversely, four members, $S a H s f A 6 b, S a H s f B 2 b, S a H s f B 2 c$, and $S a H s f C 1 b$, were down-regulated compared with their expression levels under normal conditions. Other genes, SaHsfA1b, SaHsfA4c, SaHsfA8, SaHsfB2d, and SaHsfB2f, which had high expression levels under control conditions, were also up-regulated under $\mathrm{CdCl}_{2}$ treatment. A similar phenomenon was also found in the stem and leaf (Figure 4B,C). Specifically, SaHsfB2a and SaHsfB2e were only up-regulated by $C d$ stress in the root, as were SaHsfA1a, SaHsfA1d, SaHsfB1, and SaHsfC1b in the stem, and $S a H s f B 4 b$ in the leaf. Unlike these genes, SaHsfA1b, SaHsfA4a, SaHsfA4c, SaHsfA8, and SaHsfB2f were up-regulated in all of the tested tissues.

Among the up-regulated genes, SaHsfA5 was strongly induced in the root and stem at $1 \mathrm{~h}$ after $\mathrm{Cd}$ treatment, while its expression was down-regulated in the leaf. Notably, SaHsfA4a was induced at the earlier stage of treatment $(0.5-1 \mathrm{~h})$ in the stem, and at the late stage $(6-12 \mathrm{~h})$ in the root and leaf, while $S a H s f A 4 c$, in the same class, had an opposite expression pattern. 
A

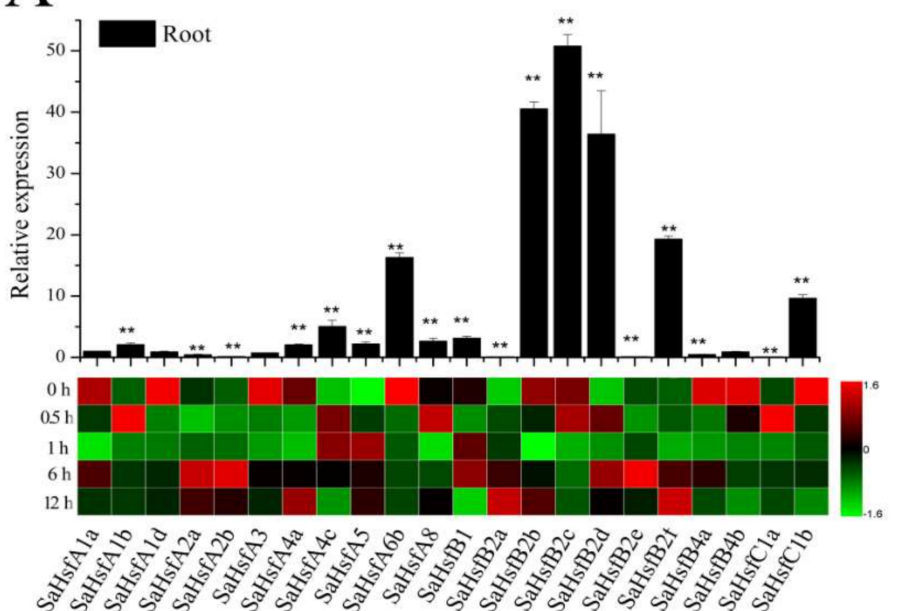

B
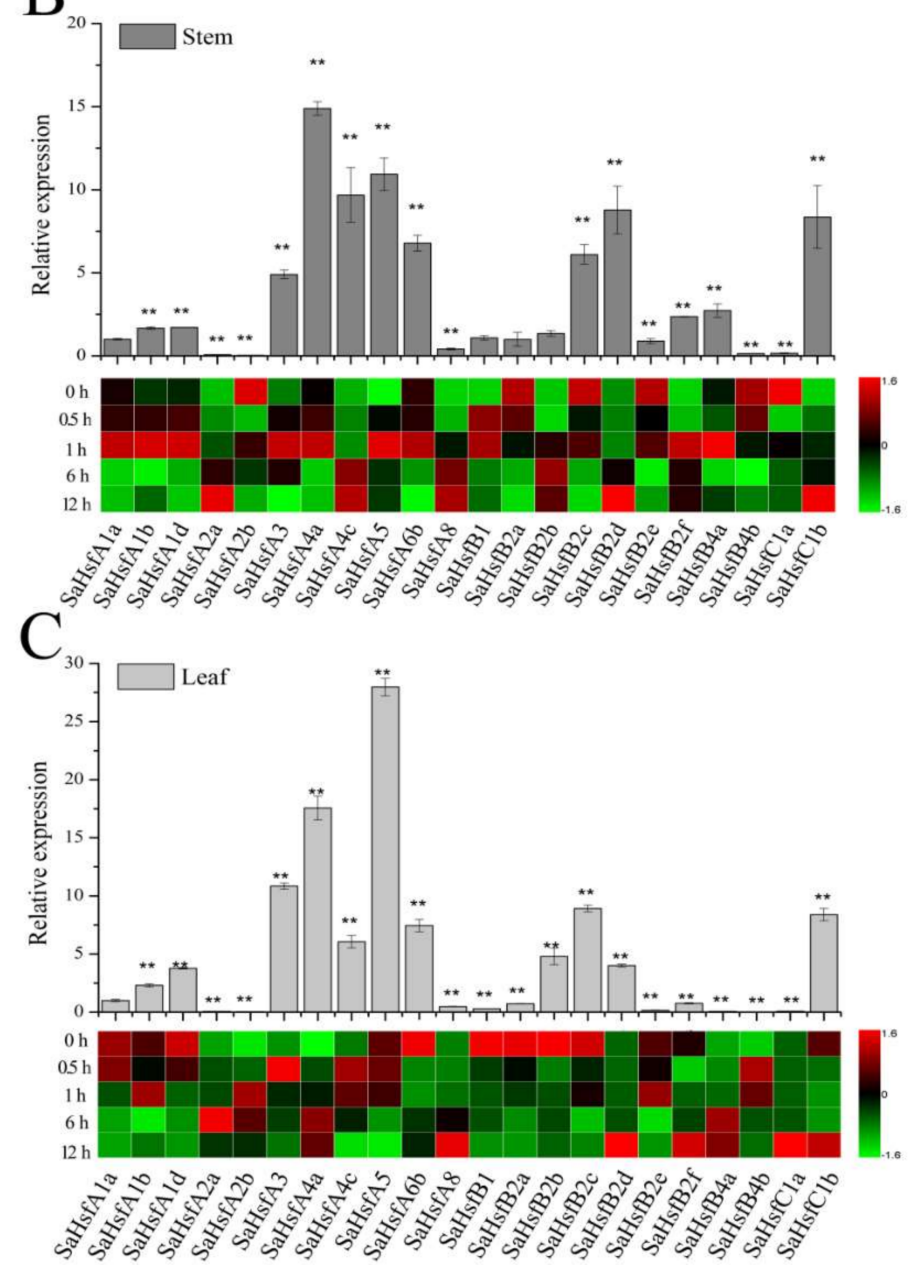

Figure 4. Expression profiles of $S a H s f s$ in different tissues under normal and cadmium (Cd) stress conditions: (A) root; (B) stem; (C) leaf. Column chart and heat map representation for the expression patterns of the $22 \mathrm{SaHsf}$ genes under normal and $\mathrm{CdCl}_{2}$ treatment, respectively. The normalized mRNA levels of SaHsfA1a (y-axis "Relative mRNA expression") were set arbitrarily to 1 in the column chart, under normal condition. The normalized mRNA levels without treatment were set arbitrarily to 1 in the heat map. Different colors correspond to log2 transformed values compared with the control $(0 \mathrm{~h})$. Green and red represent the low and high level of transcript abundance, respectively. Bars indicate means \pm standard deviations (SDs) of at least three independent biological experiments. ${ }^{* *}-p<0.01$. 


\subsection{SaHsfs and Their Downstream Genes, SaHsps, Exhibited Similar Expression Patterns}

The expression patterns of the SaHsps, as downstream genes of SaHsfs, were tested and compared with those of the SaHsfs using qRT-PCR. There were three different expression profiles that were observed among the SaHsfs and SaHsps (Figure 5). At the earlier stage of the Cd treatment (0.5-1 h), SaHsp70 was up-regulated in the root together with SaHsfA1b, SaHsfA8, SaHsfB2c, and SaHsfC1a, while $S a H s f A 1 b, S a H s f A 2 b$, and $S a H s f B 2 e$ were up-regulated in the leaf, along with $S a H s p 18.1$ and SaHsp101 (Figure 5A,G). There were 10 SaHsf genes (including SaHsfA1, SaHsfA3, SaHsfA4a, SaHsfA5, $S a H s f A 6 b, S a H s f B 1, S a H s f B 2 f$, and $S a H s f B 4 a)$ that had similar expression patterns to SaHsp18.1 and SaHsp101, with a peak in the stem after $1 \mathrm{~h}$ of $\mathrm{Cd}$ treatment (Figure 5D). After $6 \mathrm{~h}$ of treatment, SaHsp101, SaHsp22/SaHsp26.5/SaHsp70, and SaHsp22/SaHsp26.5 reached the highest expression levels in the root, stem, and leaf, respectively. Similar expression profiles were also found among other SaHsfs, namely, SaHsfA2a, SaHsfA2b, SaHsfA4a, SaHsfB1, SaHsfB2b, SaHsfB2e, and SaHsfB4a (Figure 5B,E,H). A continuously increasing expression pattern was considered to be the third profile in the root, stem, and leaf (Figure 5C,F,I). SaHsfA4a, SaHsfA4c, SaHsfA5, SaHsfA8, SaHsfB2a, SaHsfB2d, SaHsfB2f, SaHsfC1a, and $S a H s f C 1 b$ indicated this expression pattern, as did the SaHsp18.1, SaHsp22, and SaHsp90 genes (Figure 5C,F,I). In addition, the SaHsp genes exhibited higher expression levels than the SaHsf genes in S. alfredii under Cd stress (Figure 5).

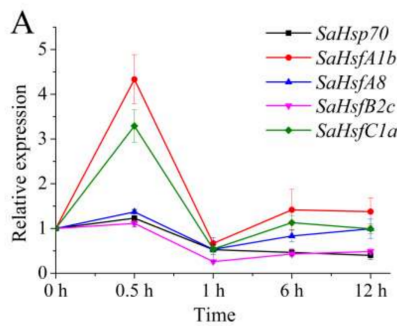

D

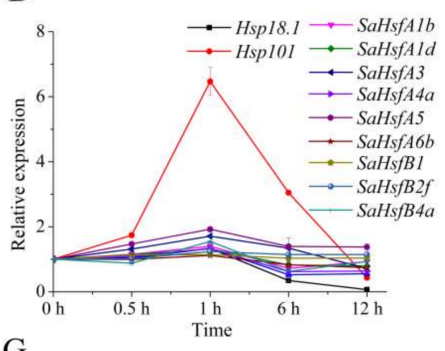

G

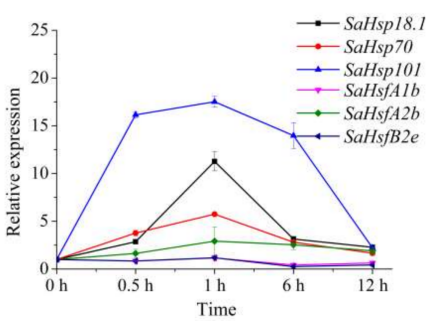

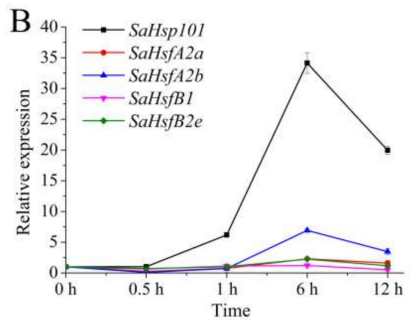

E

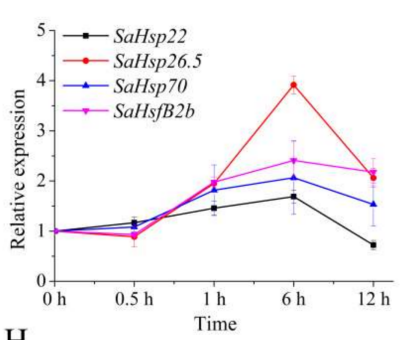

$\mathrm{H}$

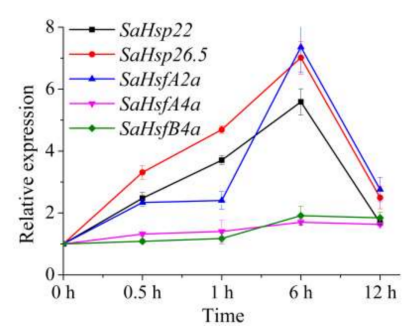

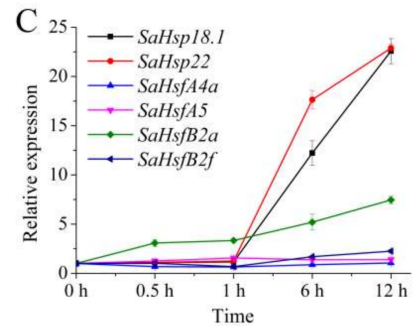

F
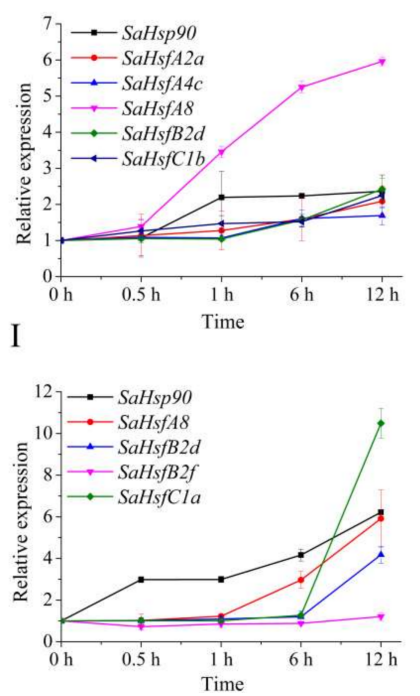

Figure 5. The expression profiles of SaHsps and SaHsfs in different tissues under Cd stress: The normalized mRNA levels without treatment were set arbitrarily to 1. (A-C) three similar expression patterns between SaHsps and SaHsfs in the root; (D-F) three similar expression patterns between SaHsps and SaHsfs in the stem; (G-I) three similar expression patterns between SaHsps and SaHsfs in the leaf. Bars indicate means \pm standard deviations (SDs) of at least three independent biological experiments.

\subsection{Class SaHsfA4' Expression Enhanced Cd Tolerance in Yeast}

To analyze the function of the class SaHsfA4 members (SaHsfA4a and SaHsfA4c), the genes were expressed in a Saccharomyces cerevisiae yeast mutant strain ( $\Delta y c f 1)$ that was susceptible to excessive 
Cd. The verified recombinant plasmids (pYES-DEST52-SaHsfA4a and pYES-DEST52-SaHsfA4c) and empty vector (pYES2.0) were transformed into $\triangle y c f 1$ cells, which were then grown in a synthetic-galactose-uracil (SG-U) medium. $\triangle y c f 1$ cells expressing SaHsfA4a and SaHsfA4c exhibited remarkably enhanced growth status when compared with $\triangle y c f 1$ cells transformed with the empty vector $(\triangle y c f 1+\mathrm{EV})$ on SG-U medium with $\mathrm{CdCl}_{2}$ (Figure 6A,B). Additionally, the metal content was measured in the yeast cells expressing SaHsfA4a, SaHsfA4c, or the empty vector, which were grown in the presence of $\mathrm{Cd}$ for about $96 \mathrm{~h}$. A significantly increased accumulation of $\mathrm{Cd}$ was observed in the yeast cells expressing SaHsfA4a or SaHsfA4c compared with the control (Figure 6C). Furthermore, yeast cells (AH109) containing either the control (pGBKT7) or fusion plasmids (pGBKT7-SaHsfA4a and pGBKT7-SaHsfA4c) grew well on SD/Trp ${ }^{-}$plates. Only the yeast cells containing fusion plasmids could grow on $\mathrm{SD} / \mathrm{Trp}^{-} \mathrm{His}^{-}$plates and they turned blue in the presence of $\mathrm{X}-\alpha$-gal (Supplementary Figure S1). These results suggested that $S a H s f A 4 a$ and $S a H s f A 4 c$ were transcription activators and could activate the expression of the GAL4 upstream activation sequence-driven LacZ reporter gene.

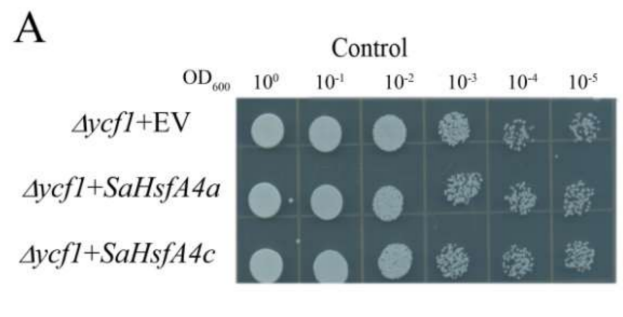

$\mathrm{B}$

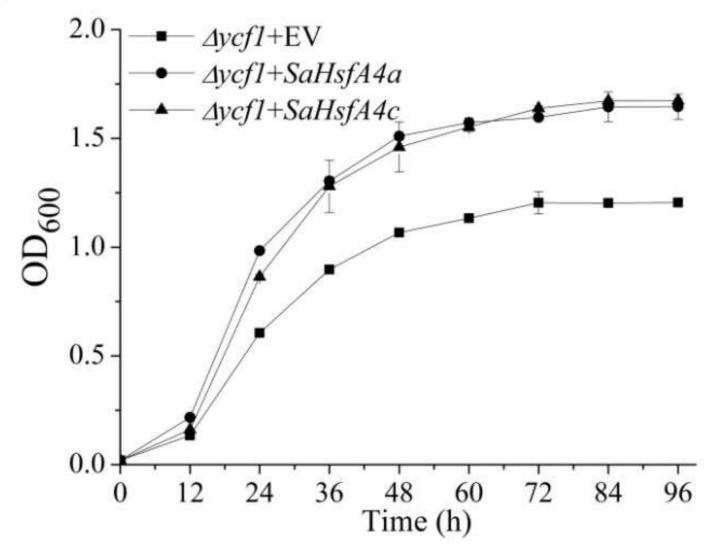

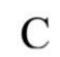

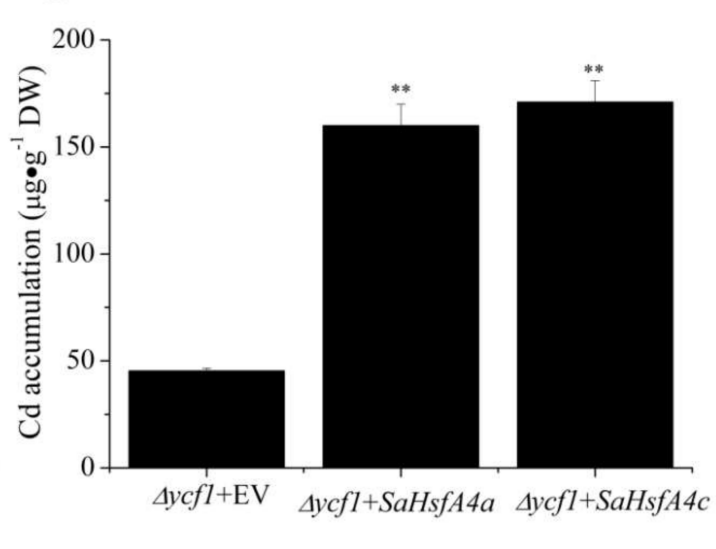

Figure 6. Overexpression of $S a H s f A 4 a$ or $S a H s f A 4 c$ increases the Cd tolerance and accumulation in yeast. (A) the growth of $\triangle y c f 1$ yeast mutants transformed with the empty vector pYES2.0 or with pYES-DEST52 harboring SaHsfA4a or SaHsfA4c; (B) time-dependent growth of yeast strains in synthetic-galactose-uracil (SG-U) liquid medium supplemented with $15 \mu \mathrm{M} \mathrm{CdCl}_{2}$; and (C) the accumulation of $\mathrm{Cd}$ in $\Delta y c f 1$ yeast cells. Bars indicate means \pm standard deviations (SDs) of at least three independent biological experiments. Two asterisks indicate a significant difference at $p<0.01$ from the $\Delta y c f 1+\mathrm{EV}$.

\section{Discussion}

The Hsf family has been researched in many plant species [2,21,35-37], but little information is available on the characteristics of the Hsfs in S. alfredii under heavy metal stress. In this study, $22 \mathrm{Hsf}$ genes were screened and identified in S. alfredii, based on transcriptome data (Table 1). The phylogenetic analysis indicated that the $S a H s f s$ could be divided into three major groups corresponding with those in A. thaliana and O. sativa (Figure 1), which was consistent with previous reports [13-15,18]. Although the total number of SaHsf genes was similar to those in other plant species, such as A. thaliana, O. sativa, P. trichocarpa, and Z. mays (Table 2), the sizes of some subclasses in S. alfredii differed from those in other 
species. For instance, no subclass A7 and A9 members were found. Conversely, the number of genes in class B2 was greater than those in other species, suggesting that gene loss and gene duplication events had occurred at the different stages of the evolutionary process, resulting in Hsf diversity [13,38]. Each class of SaHsfs shared similar motifs (Figure 2), implying that the Hsf genes were evolutionarily well conserved or had similar regulatory functions in S. alfredii.

Previous studies on Hsfs had focused on their roles in responses to abiotic stresses such as heat, salt, drought, cold, and hormones $[3,12,17,23,24,39]$. Only a few reports had indicated that some Hsf members played critical roles in enhancing $C d$ tolerance $[25,26]$. Here, a single genotype of $S$. alfredii seedlings was asexually propagated and used in $\mathrm{CdCl}_{2}$ treatment. We examined the expression profiles of the SaHsf genes in different tissues after $400 \mu \mathrm{M} \mathrm{CdCl}_{2}$ treatment. More than half of the SaHsf genes were responsive to heavy metal (Cd) stress, which may be due to the properties of the experimental material (S. alfredii) as a $\mathrm{Cd} / \mathrm{Zn} / \mathrm{Pb}$ co-hyperaccumulator. Under normal conditions, the genes SaHsfA1b, SaHsfA4a, SaHsfA4c, SaHsfA5, SaHsfA6b, SaHsfB2c, SaHsfB2d, and SaHsfC1b were expressed in all tested tissues, while $S a H s f A 8, S a H s f B 1$, and $S a H s f B 2 f$ transcripts were detected primarily in the root and $S a H s f B 4 a$ transcripts in the stem (Figure 4), suggesting that the expression of these Hsf family members is tissue specific. After $\mathrm{CdCl}_{2}$ treatment, $\mathrm{SaHsfB2a}$ and $\mathrm{SaHsfB2e}$ were only up-regulated in the root, $S a H s f A 1 a, A 1 d, B 1$ and $C 1 b$ in the stem, and $S a H s f B 4 b$ in the leaf. These results suggested that the Hsf family members in $S$. alfredii are induced in a tissue-specific manner under Cd stress. It was reported that overexpression of HsfA1a from S. lycopersicum L. cv Ailsa Craig enhanced plant tolerance to $\mathrm{Cd}$ [25], and that class HsfA4 genes in wheat and rice conferred $\mathrm{Cd}$ tolerance [26]. Our study found that $S a H s f A 1 b$, SaHsfA4a, and SaHsfA4c were up-regulated in all of the tested tissues under Cd stress. We also found that SaHsfA4a and SaHsfA4c had the opposite expression patterns at different stages of treatment in different tissues. SaHsfA5 expression was strongly induced in the root and stem, and reduced in the leaf under $\mathrm{Cd}$ treatment. In Arabidopsis, AtHsfA5 acted as a specific repressor of AtHsfA4 and was considered to be an antiapoptotic factor [9,40]. Furthermore, HsfA4 and HsfA5 were found to have a close relationship in the phylogenetic tree and had similar motifs in their C terminal [40]. In our study, a similar result was observed in S. alfredii (Figure 2). However, the expression profiles of $H s f A 4$ and $H s f A 5$ were very different. Thus, their roles in heavy metal response deserve further investigation.

$H s p s$ are the downstream genes of $H s f s$ that protect plants against abiotic stress damage [40,41]. $H s f s$ are responsible for the transcription of $H s p s$, such as small heat shock proteins (sHsps), Hsp 70, and Hsp101 [7,40,42]. Hsps could be induced by various abiotic stresses, such as cold, heat, and salt [7,42-44]. Overexpression of GmHsfA1 could enhance heat tolerance by regulating downstream Hsp genes (such as GmHsp22 and GmHsp70) in transgenic soybeans [45]. It was also reported that multiple Hsp genes, including Hsp18.1, Hsp22, Hsp26.5, Hsp70, and Hsp101, were positively induced by heat treatment in the transgenic Arabidopsis and tall fescue overexpressing FaHsfA2c [42]. CmHsp70 and CmHsp90 were considered the direct target genes of CmHsfA4 in the transgenic Chrysanthemum plants, both in non-stress and salt stress conditions. In our study, all of the tested Hsp genes, including SaHsp18.1, SaHsp22, SaHsp26.5, SaHsp70, SaHsp90, and SaHsp101, were up-regulated remarkably by Cd stress in the root, stem, or leaf (Figure 5). Moreover, three similar expression patterns between SaHsps and $\mathrm{SaHsfs}$ were exhibited under $\mathrm{Cd}$ treatment. These results suggested that $\mathrm{SaHsfs}$ could regulate the expression levels of SaHsps when the plants were exposed to heavy metal stresses.

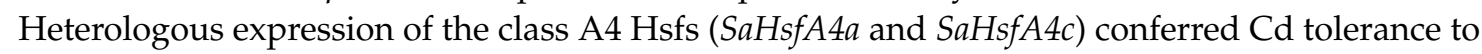
yeast (Figure 6). Previously, class A4 Hsfs had been reported as being induced by other abiotic stresses in plants. AtHsfA4a had been involved in high-light and oxidative stress responses by regulating the transcription of APX1 and ZAT12 genes [44,46]. Co-overexpression of Helianthus annuus HaHSFA4a and HaHSFA9 enhanced tolerance to dehydration and severe oxidative stress in transgenic tobacco [47]. Overexpression of the CmHsfA4 gene positively enhanced salt stress tolerance in transgenic Chrysanthemum [44]. Additionally, studies have shown that OsHsfA4a and TaHsfA4a conferred tolerance to $\mathrm{Cd}$ [26]. Here, the $\Delta y c f 1$ yeast strain harboring the fusion vectors 
(pYES-DEST52-SaHsfA4a or pYES-DEST52-SaHsfA4a) exhibited better growth than the control $(\Delta y c f 1+$ EV) (Figure 6A,B). Moreover, the transgenic yeast harboring the fusion vectors had a higher $\mathrm{Cd}$ content than the control (Figure 6C). These results indicated that the class SaHsfA4 members might have been involved in responding to the $\mathrm{Cd}$ stress, and that these genes could complement the $\mathrm{Cd}$ sensitivity in the mutant yeast strain. Additionally, transcriptional activation assay revealed SaHsfA4a and SaHsfA4c were transcription activators in yeast cells (Supplementary Figure S1). Thus, we speculated that $\mathrm{SaHsfA4a}$ and $\mathrm{SaHsfA4c}$ as transcription activators, could activate target genes to enhance $\mathrm{Cd}$ tolerance and improve $\mathrm{Cd}$ accumulation. Similarly, Shim et al. found that transgenic yeast expressing OsHsfA4a and TaHsfA4a were tolerant to $\mathrm{Cd}$, and that the yeast strain overexpressing TaHsfA4a grew better in liquid cultures supplemented with $\mathrm{CdCl}_{2}$ than the control [26].

Overall, we have presented a comprehensive analysis of the Hsf gene family members in S. alfredii as well as their expression under Cd stress. The $22 \mathrm{Hsf}$ members were phylogenetically clustered into three classes. The expression profiles of the $S a H s f s$ showed significant differences; among them, $18 \mathrm{SaHsfs}$ were found to respond to Cd stress. SaHsfA4a and SaHsfA4c exhibited transcriptional activation activities. Overexpression of the $S a H s f A 4 a$ or $S a H s f A 4 c$ gene enhanced tolerance to Cd stress in transgenic yeast. Our results will be beneficial for elucidating the mechanism of heavy metal stresses regulation by SaHsfs.

\section{Methods}

\subsection{Plant Materials and Stress Treatments}

A hyperaccumulating ecotype of S. alfredii, collected from an old $\mathrm{Pb} / \mathrm{Zn}$ mine in Quzhou City, Zhejiang Province, China, was first identified by Yang et al. [27].

The seedlings were water-cultivated in an artificial climate chamber for long-day treatment ( $16 \mathrm{~h}$ light $/ 8 \mathrm{~h}$ dark cycle) at $25^{\circ} \mathrm{C}$. The $\mathrm{S}$. alfredii seedlings from a single genotype were asexually propagated and grown in a half-strength Hoagland solution for about three weeks. For heavy metal stress treatment, the roots were dipped into a solution containing $400 \mu \mathrm{M} \mathrm{CdCl}_{2}$, while a set of control seedlings were similarly cultured in a half-strength Hoagland solution. The treated root, stem, and leaf were then harvested at $0,0.5,1,6$, and $12 \mathrm{~h}$. All of the samples from the three biological replicates were frozen at $-80^{\circ} \mathrm{C}$ for subsequent analysis.

\subsection{Identification of SaHsfs in S. alfredii}

The coding and peptide sequences of candidate $S a H s f s$ were searched in the transcriptome database of S. alfredii [34]. An HMM search (http://www.ebi.ac.uk/Tools/hmmer/search/hmmsearch) was conducted to identify and retrieve possible Hsfs containing DBD (Pfam: PF00447) domains in S. alfredii. Some UniGenes that were produced by the transcriptome sequencing did not contain the full coding regions of the genes. Thus, the putative S. alfredii SaHsfs with incomplete coding sequences were conducted and cloned to obtain the full coding regions through a homologous cloning strategy. The Hsf homologs in A. thaliana and O. sativa were obtained from TAIR (https:/ / www.arabidopsis.org/) and the TIGR-Rice Genome Annotation Project (http:/ / rice.plantbiology.msu.edu/), respectively (Supplementary Table S1). All of the candidate genes were confirmed by SMART (http:/ / smart.embl-heidelberg.de/) and Pfam (http:/ / pfam.xfam.org/search). In addition, all of the SaHsf genes were named according to the orthologous genes in A. thaliana. The molecular weights $(\mathrm{kDa})$ and isoelectric points $(\mathrm{pI})$ of the Hsfs were calculated by the ExPASy program (http://web.expasy.org/compute_pi) and DNAMAN software.

\subsection{Multiple Sequence Alignment, Conserved Motif and Domain Prediction}

ClustalX was used to align the amino acid sequences of all of the candidate S. alfredii Hsf proteins. Subsequently, the result was edited manually with GeneDoc. MEME (http:/ / meme.sdsc.edu) was used to identify the motifs in the candidate sequences and was run locally with the following parameters: number of repetitions $=$ any; maximum number of motifs $=25$; and optimum motif width $=6-100$ 
residues. The distribution diagram of the motifs was edited with the IBS software [48]. HEATSTER (http:/ / www.cibiv.at/services/hsf/) online tools were used to analyze the SaHsf proteins' typical functional structural domains [7].

\subsection{Phylogenetic Analysis and Classification of SaHsf Genes}

The amino acid sequences of the Hsf proteins from S. alfredii, Arabidopsis and rice were aligned using the ClustalX program. Subsequently, the MEGA 6.0 software was used to construct an unrooted neighbor-joining phylogenetic tree with a bootstrap test that was replicated 1000 times [49]. The SaHsf genes were assigned to different groups based on the phylogenetic tree and the HEATSTER predictions.

\subsection{Total RNA Isolation and Expression Analysis}

The total RNA was isolated from root, stem, and leaf using a total RNA kit (NORGEN, Thorold, ON, Canada) and treated with DNase I (TaKaRa, Dalian, China) to digest any genomic DNA. First-strand cDNA was generated using PrimeScript ${ }^{\mathrm{TM}}$ RT Master Mix (TaKaRa, Dalian, China) following the manufacturer's instructions and then stored at $-20^{\circ} \mathrm{C}$ until use.

qRT-PCR was carried out in triplicate on an Applied Biosystems 7300 Real-Time PCR System thermal cycler (Applied Biosystems, CA, USA) using SYBR ${ }^{\circledR}$ Premix Ex Taq ${ }^{\mathrm{TM}}$ reagent, (TaKaRa, Dalian, China). The primer sequences that were used in qRT-PCR are shown in Supplementary Table S2. The amplification procedure was performed according to a previous study $[33,50]$. The relative expression level of each of the SaHsf genes was calculated based on the comparative threshold cycle $\left(2^{-\Delta \Delta C T}\right)$ method. The SaUBC9 gene was used as an endogenous reference gene to normalize the threshold values (Ct) of the SaHsf and SaHsp genes [51]. The primers were designed using the online tool, Primer 3.0 (http:/ / bioinfo.ut.ee/primer3-0.4.0/primer3/), based on the non-conserved regions of the SaHsf and SaHsp genes, and were synthesized by BioSune Company (Shanghai, China).

The normalized mRNA levels of SaHsfA1a (y-axis "Relative mRNA expression") were set arbitrarily to 1 , under normal condition. To analyze the $S a H s f$ genes' relative expression under Cd stress, the normalized mRNA levels without treatment (y-axis "Relative mRNA expression") were also set arbitrarily to 1 . Subsequently, the z-score method was used to normalize the original expression levels of the SaHsfs, in order to draw a heat-map with HemI (The Cuckoo Workgroup, Wuhan, China). The absolute signal intensity ranged from -1.6 to +1.6 , and the color scale (green and red) represented the expression values (low and high level). Bars indicated means \pm standard deviations (SDs) of at least three of the independent biological experiments.

\subsection{Heterologous Expression of SaHsfA4a and SaHsfA4c in Yeast}

The specific primers SaHsfA4a-F/R and SaHsfA4c-F/R (Supplementary Table S2) were used to amplify the open reading frames in S. alfredii. The purified PCR products were then cloned into the Gateway entry vector pDONR222 (Invitrogen, Carlsbad, USA), and recombined into pYES-DEST52 to generate pYES-DEST52-SaHsfA4a and pYES-DEST52-SaHsfA4c, respectively. The $\Delta y c f 1$ strain was transformed with each construct, using the lithium acetate method [33,52]. The empty pYES2.0 vector was used as control. To perform the $\mathrm{Cd}$ tolerance test, the transgenic yeast lines were grown up to $\mathrm{OD}_{600}=1$, and then serially diluted $\left(\mathrm{OD}_{600}=10^{0}, 10^{-1}, 10^{-2}, 10^{-3}, 10^{-4}\right.$, and $\left.10^{-5}\right)$, spotted on SG-U agar plates supplemented with 0,15 , and $30 \mu \mathrm{M} \mathrm{CdCl}_{2}$, and incubated at $28{ }^{\circ} \mathrm{C}$ for 3 days $[33,50]$. Additionally, the relative growth of transformants was determined by measuring $\mathrm{OD}_{600}$ at $12 \mathrm{~h}$ intervals. For the Cd-uptake assay, yeast cells that were transformed with the empty, pYES-DEST52-SaHsfA4a or pYES-DEST52-SaHsfA4c vector were grown at $28{ }^{\circ} \mathrm{C}$ on SG-U, supplemented with $15 \mu \mathrm{M} \mathrm{CdCl}_{2}$ for $96 \mathrm{~h}$, and finally, the $\mathrm{Cd}$ accumulation was measured [50].

\subsection{Transcriptional Activation Activity Assay of Two SaHsfA4 Members in Yeast}

Transcriptional activation vectors were constructed and specific primers were designed (Supplementary Table S2) using a GBclonart Cloning Kit (GBI, Suzhou, China). The complete coding 
sequences of SaHsfA4a and SaHsfA4c were amplified by PCR using specific primers. The PCR products were cloned into the pGBKT7 vector to create pGBKT7-SaHsfA4a and pGBKT7-SaHsfA4c. The sequence-verified plasmids were transformed in the yeast strain AH109, using the lithium acetate method [52]. The transformed strains were confirmed by PCR and sequencing, and then plated on a $\mathrm{SD} / \mathrm{Trp}^{-}, \mathrm{SD} / \mathrm{Trp}^{-} \mathrm{His}^{-}$, or SD/Trp ${ }^{-} \mathrm{His}^{-}+\mathrm{X}-\alpha$-gal medium. Transcription activation activity was evaluated according to the growth status of the yeast cells after incubating the plates at $28^{\circ} \mathrm{C}$ for 3 days.

\section{Conclusions}

In the present study, a comprehensive analysis of the Hsf family was performed, including phylogenetic, conserved domain, and motif analyses and expression profiling, under $\mathrm{Cd}$ stress based on transcriptome sequencing. A total of $22 \mathrm{Hsf}$ members were identified from S. alfredii using bioinformatics. They were phylogenetically clustered into three classes, namely, SaHsf $A, B$, and $C$, according to their structural and phylogenetic features. According to the phylogenetic tree, the majority of the subfamilies contained members from Arabidopsis, rice, and S. alfredii, suggesting that the functions of most Hsfs had been conserved during the evolutionary progress. The largest subgroup, class A, included $11 \mathrm{Hsf}$ members, followed by class B with 9 members, while class C contained only two members. Each class shared similar motifs, indicating that the $H s f s$ were markedly conserved during the evolution of S. alfredii. In addition, expression analysis indicated that the Hsfs and Hsps in S. alfredii may play important roles in responses to Cd stress. Moreover, class SaHsfA4 members exhibited transcriptional activation activities and positively regulated $\mathrm{Cd}$ stress tolerance and accumulation in yeast. Our results provide a solid foundation for further functional dissection of the SaHsf family, and will improve our understanding of the characteristics of SaHsf genes in hyperaccumulating species.

Supplementary Materials: Supplementary materials can be found at http:/ /www.mdpi.com/1422-0067/19/4/ $1216 /$ s1.

Acknowledgments: This work was supported by the National Nonprofit Institute Research Grant of CAF (No. RISF2016002, TGB2013008 and RISF2014010), the National Natural Science Foundation of China (No. 31200465), and the National High Technology Research and Development Program of China (No. 2013AA102701-3).

Author Contributions: Shuang-Shuang Chen contributed to data analysis and manuscript preparation. Shuang-Shuang Chen and Ren-Ying Zhuo planned and designed the research. Shuang-Shuang Chen and Xiao-Jiao Han performed the experiments, wrote the manuscript and coordinated its revision. Jing Jiang and Yun-Xing Zhang helped in sample preparation and data collection. All authors read and provided helpful discussions, and approved the final version.

Conflicts of Interest: The authors declare no conflict of interest.

\begin{tabular}{|c|c|}
\hline аa & amino acid \\
\hline ABA & abscisic acid \\
\hline bp & base pair \\
\hline $\mathrm{kDa}$ & kilodalton \\
\hline $\mathrm{pI}$ & isoelectric points \\
\hline qRT-PCR & quantitative real-time polymerase chain reaction \\
\hline SG-U & synthetic-galactose-uracil \\
\hline$X$ - $\alpha$-gal & 5-bromo-4-chloro-3-indoxyl- $\alpha$-D-galacto-pyranoside \\
\hline SD & synthetic dropout nutrient medium \\
\hline $\mathrm{SD} / \operatorname{Trp}^{-}$ & SD medium lacking tryptophan \\
\hline $\mathrm{SD} / \operatorname{Trp}^{-} \mathrm{His}^{-}$ & SD medium lacking tryptophan and histidine \\
\hline
\end{tabular}

\section{References}

1. Wang, M.; Vannozzi, A.; Wang, G.; Liang, Y.H.; Tornielli, G.B.; Zenoni, S.; Cavallini, E.; Pezzotti, M.; Cheng, Z.M. Genome and transcriptome analysis of the grapevine (Vitis vinifera L.) WRKY gene family. Hortic. Res. 2014, 1, 14016. [CrossRef] [PubMed] 
2. Guo, M.; Liu, J.H.; Ma, X.; Luo, D.X.; Gong, Z.H.; Lu, M.H. The plant heat stress transcription factors (Hsfs): Structure, regulation, and function in response to abiotic stresses. Front. Plant Sci. 2016, 7, 114. [CrossRef] [PubMed]

3. Wang, X.M.; Shi, X.; Chen, S.Y.; Ma, C.; Xu, S.B. Evolutionary origin, gradual accumulation and functional divergence of heat shock factor gene family with plant evolution. Front. Plant Sci. 2018, 9, 71. [CrossRef] [PubMed]

4. Akerfelt, M.; Morimoto, R.I.; Sistonen, L. Heat shock factors: Integrators of cell stress, development and lifespan. Nat. Rev. Mol. Cell Biol. 2010, 11, 545-555. [CrossRef] [PubMed]

5. Wang, R.H.; Yi, M.; Xu, L.; Zhu, X.W.; Wang, Y.; Guo, J.; Liu, L.W. Genome-wide characterization of differentially expressed genes provides insights into regulatory network of heat stress response in radish (Raphanus sativus L.). Funct. Integr. Genom. 2018, 1-15. [CrossRef] [PubMed]

6. Gomez-pastor, R.; Burchfiel, E.T.; Thiele, D.J. Regulation of heat shock transcription factors and their roles in physiology and disease. Nat. Rev. Mol. Cell Biol. 2018, 19, 4-19. [CrossRef] [PubMed]

7. Scharf, K.D.; Berberich, T.; Ebersberger, I.; Nover, L. The plant heat stress transcription factor (Hsf) family: Structure, function and evolution. Biochim. Biophys. Acta 2012, 1819, 104-119. [CrossRef] [PubMed]

8. Yamaguchi-Shinozaki, K.; Shinozaki, K. Transcriptional regulatory networks in cellular responses and tolerance to dehydration and cold stresses. Annu. Rev. Plant Biol. 2006, 57, 781-803. [CrossRef] [PubMed]

9. Baniwal, S.K.; Bharti, K.; Chan, K.Y.; Fauth, M.; Ganguli, A.; Kotak, S.; Mishra, S.K.; Nover, L.; Port, M.; Scharf, K.D. Heat stress response in plants: A complex game with chaperones and more than twenty heat stress transcription factors. J. Biosci. (Bangalore) 2004, 29, 471-487. [CrossRef]

10. Nakai, A. Heat Shock Factor; Springer: Tokyo, Japan, 2016.

11. Sémon, M.; Wolfe, K.H. Consequences of genome duplication. Curr. Opin. Genet. Dev. 2007, 17, 505-512. [CrossRef] [PubMed]

12. Tang, R.; Zhu, W.; Song, X.; Lin, X.; Cai, J.; Wang, M.; Yang, Q. Genome-wide identification and function analyses of heat shock transcription factors in potato. Front. Plant Sci. 2016, 7, 490. [CrossRef] [PubMed]

13. Guo, J.; Wu, J.; Ji, Q.; Wang, C.; Luo, L.; Yuan, Y.; Wang, Y.; Wang, J. Genome-wide analysis of heat shock transcription factor families in rice and Arabidopsis. J. Genet. Genom. 2008, 35, 105-118. [CrossRef]

14. Wang, F.; Dong, Q.; Jiang, H.; Zhu, S.; Chen, B.; Xiang, Y. Genome-wide analysis of the heat shock transcription factors in Populus trichocarpa and Medicago truncatula. Mol. Biol. Rep. 2012, 39, 1877-1886. [CrossRef] [PubMed]

15. Chung, E.; Kim, K.M.; Lee, J.H. Genome-wide analysis and molecular characterization of heat shock transcription factor family in Glycine max. J. Genet. Genom. 2013, 40, 127-135. [CrossRef] [PubMed]

16. Lin, Y.X.; Jiang, H.Y.; Chu, Z.X.; Tang, X.L.; Zhu, S.W.; Cheng, B.J. Genome-wide identification, classification and analysis of heat shock transcription factor family in maize. BMC Genom. 2011, 12, 76. [CrossRef] [PubMed]

17. Xue, G.P.; Sadat, S.; Drenth, J.; McIntyre, C.L. The heat shock factor family from Triticum aestivum in response to heat and other major abiotic stresses and their role in regulation of heat shock protein genes. J. Exp. Bot. 2014, 65, 539-557. [CrossRef] [PubMed]

18. Zhang, J.; Liu, B.B.; Li, J.B.; Zhang, L.; Wang, Y.; Zheng, H.Q.; Lu, M.Z.; Chen, J. Hsf and Hsp gene families in Populus: Genome-wide identification, organization and correlated expression during development and in stress responses. BMC Genom. 2015, 16, 181. [CrossRef] [PubMed]

19. Nover, L.; Bharti, K.; Döring, P.; Mishra, S.K.; Ganguli, A.; Scharf, K.D. Arabidopsis and the heat stress transcription factor world: How many heat stress transcription factors do we need? Cell Stress Chaperones 2001, 6, 177-189. [PubMed]

20. Wang, C.; Zhang, Q.; Shou, H.X. Identification and expression analysis of OsHsfs in rice. J. Zhejiang Univ. Sci. B 2009, 10, 291-300. [CrossRef] [PubMed]

21. Chauhan, H.; Khurana, N.; Agarwal, P.; Khurana, P. Heat shock factors in rice (Oryza sativa L.): Genome-wide expression analysis during reproductive development and abiotic stress. Mol. Genet. Genom. 2011, 286, 171-187. [CrossRef] [PubMed]

22. Miller, G.; Mittler, R. Could heat shock transcription factors function as hydrogen peroxide sensors in plants? Ann. Bot. 2006, 98, 279-288. [CrossRef] [PubMed]

23. Yokotani, N.; Ichikawa, T.; Kondou, Y.; Matsui, M.; Hirochika, H.; Iwabuchi, M.; Oda, K. Expression of rice heat stress transcription factor OsHsfA2e enhances tolerance to environmental stresses in transgenic Arabidopsis. Planta 2008, 227, 957-967. [CrossRef] [PubMed] 
24. Huang, Y.C.; Niu, C.Y.; Yang, C.R.; Jinn, T.L. The heat stress factor HsfA6b connects ABA signaling and ABA-mediated heat responses. Plant Physiol. 2016, 172, 1182-1199. [CrossRef] [PubMed]

25. Cai, S.Y.; Zhang, Y.; Xu, Y.P.; Qi, Z.Y.; Li, M.Q.; Ahammed, G.J.; Xia, X.J.; Shi, K.; Zhou, Y.H.; Reiter, R.J. HsfA1a upregulates melatonin biosynthesis to confer cadmium tolerance in tomato plants. J. Pineal Res. 2017, 62, e12387. [CrossRef] [PubMed]

26. Shim, D.; Hwang, J.U.; Lee, J.; Lee, S.; Choi, Y.; An, G.; Martinoia, E.; Lee, Y. Orthologs of the class A4 heat shock transcription factor HsfA4a confer cadmium tolerance in wheat and rice. Plant Cell 2009, 21, 4031-4043. [CrossRef] [PubMed]

27. Yang, X.E.; Long, X.X.; Ni, W.Z.; Fu, C.X. Sedum alfredii H: A new Zn hyperaccumulating plant first found in China. Chin. Sci. Bull. 2002, 47, 1634-1637. [CrossRef]

28. Sun, Q.; Ye, Z.H.; Wang, X.R.; Wong, M.H. Cadmium hyperaccumulation leads to an increase of glutathione rather than phytochelatins in the cadmium hyperaccumulator Sedum alfredii. J. Plant Physiol. 2007, 164, 1489-1498. [CrossRef] [PubMed]

29. Lu, L.L.; Tian, S.K.; Yang, X.E.; Wang, X.C.; Brown, P.H.; Li, T.Q.; He, Z.L. Enhanced root-to-shoot translocation of cadmium in the hyperaccumulating ecotype of Sedum alfredii. J. Exp. Bot. 2008, 59, 3203-3213. [CrossRef] [PubMed]

30. Tian, S.K.; Xie, R.H.; Wang, H.X.; Hu, Y.; Ge, J.; Liao, X.C.; Gao, X.Y.; Brown, P.H; Lin, X.Y.; Lu, L.L. Calcium deficiency triggers phloem remobilization of cadmium in a hyperaccumulating species. Plant Physiol. 2016, 172, 2300-2313. [CrossRef] [PubMed]

31. Zhang, J.; Zhang, M.; Tian, S.K.; Lu, L.L.; Shohag, M.J.I.; Yang, X.E. Metallothionein 2 (SaMT2) from Sedum alfredii Hance confers increased Cd tolerance and accumulation in yeast and tobacco. PLoS ONE 2014, 9, e102750. [CrossRef] [PubMed]

32. Li, Z.; Han, X.J.; Song, X.X.; Zhang, Y.X.; Jiang, J.; Han, Q.; Liu, M.Y.; Qiao, G.R.; Zhuo, R.Y. Overexpressing the Sedum alfredii $\mathrm{Cu} / \mathrm{Zn}$ superoxide dismutase increased resistance to oxidative stress in transgenic Arabidopsis. Front. Plant Sci. 2017, 8, 1010. [CrossRef] [PubMed]

33. Liu, M.Y.; Qiu, W.M.; He, X.L.; Zheng, L.; Song, X.X.; Han, X.J.; Jiang, J.; Qiao, G.R.; Sang, J.; Liu, M.Q.; et al. Functional characterization of a gene in Sedum alfredii Hance resembling rubber elongation factor endowed with functions associated with cadmium tolerance. Front. Plant Sci. 2016, 7, 965. [CrossRef] [PubMed]

34. Han, X.J.; Yin, H.F.; Song, X.X.; Zhang, Y.X.; Liu, M.Y.; Sang, J.; Jiang, J.; Li, J.H.; Zhuo, R.Y. Integration of small RNAs, degradome and transcriptome sequencing in hyperaccumulator Sedum alfredii uncovers a complex regulatory network and provides insights into cadmium phytoremediation. Plant Biotechnol. J. 2016, 14, 1470-1483. [CrossRef] [PubMed]

35. Chidambaranathan, P.; Jagannadham, P.T.K.; Satheesh, V.; Kohli, D.; Basavarajappa, S.H.; Chellapilla, B.; Kumar, J.; Jain, P.K.; Srinivasan, R. Genome-wide analysis identifies Chickpea (Cicer arietinum) heat stress transcription factors $(H s f s)$ responsive to heat stress at the pod development stage. J. Plant Res. 2017, 1-18. [CrossRef] [PubMed]

36. Guo, M.; Lu, J.P.; Zhai, Y.F.; Chai, W.G.; Gong, Z.H.; Lu, M.H. Genome-wide analysis, expression profile of heat shock factor gene family (CaHsfs) and characterisation of CaHsfA2 in pepper (Capsicum annuum L.). BMC Plant Biol. 2015, 15, 151. [CrossRef] [PubMed]

37. Jiang, Y.L.; Zheng, Q.Q.; Chen, L.; Liang, Y.N.; Wu, J.D. Ectopic overexpression of maize heat shock transcription factor gene $\mathrm{ZmHsf04}$ confers increased thermo and salt-stress tolerance in transgenic Arabidopsis. Acta Physiol. Plant. 2018, 40, 9. [CrossRef]

38. Lin, Z.; Kong, H.; Nei, M.; Ma, H. Origins and evolution of the recA/RAD51 gene family: Evidence for ancient gene duplication and endosymbiotic gene transfer. Proc. Natl. Acad. Sci. USA 2006, 103, 10328-10333. [CrossRef] [PubMed]

39. Liao, W.Y.; Lin, L.F.; Jheng, J.L.; Wang, C.C.; Yang, J.H.; Chou, M.L. Identification of heat shock transcription factor genes involved in thermotolerance of octoploid cultivated strawberry. Int. J. Mol. Sci. 2016, 17, 2130. [CrossRef] [PubMed]

40. von Koskull-Doring, P.; Scharf, K.D.; Nover, L. The diversity of plant heat stress transcription factors. Trends Plant Sci. 2007, 12, 452-457. [CrossRef] [PubMed]

41. Hua, J. From freezing to scorching, transcriptional responses to temperature variations in plants. Curr. Opin. Plant Biol. 2009, 12, 568-573. [CrossRef] [PubMed] 
42. Wang, X.Y.; Huang, W.L.; Liu, J.; Yang, Z.M.; Huang, B.R. Molecular regulation and physiological functions of a novel FaHsfA2c cloned from tall fescue conferring plant tolerance to heat stress. Plant Biotechnol. J. 2017, 15, 237-248. [CrossRef] [PubMed]

43. Cao, F.L.; Cheng, H.; Cheng, S.Y.; Li, L.L.; Xu, F.; Yu, W.W.; Yuan, H.H. Expression of selected ginkgo biloba heat shock protein genes after cold treatment could be induced by other abiotic stress. Int. J. Mol. Sci. 2012, 13, 5768-5788. [CrossRef] [PubMed]

44. Li, F.; Zhang, H.R.; Zhao, H.S.; Gao, T.W.; Song, A.P.; Jiang, J.F.; Chen, F.D.; Chen, S.M. Chrysanthemum $\mathrm{C} m \mathrm{HsfA} 4$ gene positively regulates salt stress tolerance in transgenic chrysanthemum. Plant Biotechnol. J. 2017. [CrossRef] [PubMed]

45. Zhu, B.G.; Ye, C.J.; Lu, H.Y.; Chen, X.J.; Chai, G.H.; Chen, J.N.; Wang, C. Identification and characterization of a novel heat shock transcription factor gene, GmHsfA1, in soybeans (Glycine max). J. Plant Res. 2006, 119, 247-256. [CrossRef] [PubMed]

46. Davletova, S.; Mittler, R. The zinc-finger protein ZAT12 plays a central role in reactive oxygen and abiotic stress signaling in Arabidopsis. Plant Physiol. 2005, 139, 847-856. [CrossRef] [PubMed]

47. Personat, J.M.; Tejedor-Cano, J.; Prieto-Dapena, P.; Almoguera, C.; Jordano, J. Co-overexpression of two heat shock factors results in enhanced seed longevity and in synergistic effects on seedling tolerance to severe dehydration and oxidative stress. BMC Plant Biol. 2014, 14, 56. [CrossRef] [PubMed]

48. Liu, W.Z.; Xie, Y.B.; Ma, J.Y.; Luo, X.T.; Nie, P.; Zuo, Z.X.; Lahrmann, U.; Zhao, Q.; Zheng, Y.Y.; Zhao, Y.; et al. IBS: An illustrator for the presentation and visualization of biological sequences. Bioinformatics 2015, 31, 3359. [CrossRef] [PubMed]

49. Tamura, K.; Stecher, G.; Peterson, D.; Filipski, A.; Kumar, S. MEGA6: Molecular evolutionary genetics analysis version 6.0. Mol. Biol. Evol 2013, 30, 2725-2729. [CrossRef] [PubMed]

50. Chen, S.S.; Han, X.J.; Fang, J.; Lu, Z.C.; Qiu, W.M.; Liu, M.Y.; Sang, J.; Jiang, J.; Zhuo, R.Y. Sedum alfredii SaNramp6 metal transporter contributes to cadmium accumulation in transgenic Arabidopsis thaliana. Sci. Rep. 2017, 7, 13318. [CrossRef] [PubMed]

51. Sang, J.; Han, X.J.; Liu, M.Y.; Qiao, G.R.; Jiang, J.; Zhuo, R.Y. Selection and validation of reference genes for real-time quantitative PCR in hyperaccumulating ecotype of Sedum alfredii under different heavy metals stresses. PLoS ONE 2013, 8, e82927. [CrossRef] [PubMed]

52. Szczypka, M.S.; Wemmie, J.A.; Moye-Rowley, W.S.; Thiele, D.J. A yeast metal resistance protein similar to human cystic fibrosis transmembrane conductance regulator (CFTR) and multidrug resistance-associated protein. J. Biol. Chem. 1994, 269, 22853-25857. [PubMed] 